\title{
State-Independent Experimental Test of Quantum Contextuality in an Indivisible System
}

\author{
C. Zu, ${ }^{1}$ Y.-X. Wang, ${ }^{1}$ D.-L. Deng, ${ }^{1,2}$ X.-Y. Chang, ${ }^{1}$ K. Liu,,${ }^{1}$ P.-Y. Hou, ${ }^{1}$ H.-X. Yang, ${ }^{1}$ and L.-M. Duan ${ }^{1,2}$ \\ ${ }^{1}$ Center for Quantum Information, IIIS, Tsinghua University, Beijing, China \\ ${ }^{2}$ Department of Physics, University of Michigan, Ann Arbor, Michigan 48109, USA
}

(Received 30 June 2012; published 8 October 2012)

\begin{abstract}
We report the first state-independent experimental test of quantum contextuality on a single photonic qutrit (three-dimensional system), based on a recent theoretical proposal [Phys. Rev. Lett. 108, 030402 (2012)]. Our experiment spotlights quantum contextuality in its most basic form, in a way that is independent of either the state or the tensor product structure of the system.
\end{abstract}

Contextuality represents a major deviation of quantum theory from classical physics [1,2]. Noncontextual realism is a pillar of the familiar world view of classical physics. In a noncontextual world, observables have predefined values, which are independent of our choices of measurements. Noncontextuality plays a role also in the derivation of Bell's inequalities, as the property of local realism therein can be seen as a special form of noncontextuality, where the independence of the measurement context is enforced by the no-signalling principle [3-6]. In an attempt to save the noncontextuality of the classical world view, noncontextual hidden variable theories have been proposed as an alternative to quantum mechanics. In these theories, the outcomes of measurements are associated to hidden variables, which are distributed according to a joint probability distribution. However, the celebrated KochenSpecker theorem [1-4] showed that noncontextual hidden variable theories are incompatible with the predictions of quantum theory. The original Kochen-Specker theorem is presented in the form of a logical contradiction, which is conceptually striking, but experimentally unfriendly: The presence of unavoidable experimental imperfections motivated a debate on whether or not the noncontextual features highlighted by the Kochen-Specker (KS) theorem can be actually tested in experiments [7,8]. As a result of the debate, new Bell-type inequalities have been proposed in the recent years, with the purpose of pinpointing the contextuality of quantum mechanics in an experimentally testable way. These inequalities are generally referred to as the $K S$ inequalities [5]. Violation of the KS inequalities confirms quantum contextuality and rules out the noncontextual hidden variable theory. Different from the Bell inequality tests, violation of the KS inequality can be achieved independently of the state of quantum systems $[1,2,5,6]$, showing that the conflict between quantum theory and noncontextual realism resides in the structure of quantum mechanics instead of particular quantum states. The KS inequalities have been tested in experiments for two qubits, with ions [9], photons [10,11], neutrons [12], or an ensemble nuclear magnetic resonance system [13]. A single qutrit represents the simplest system where it is possible to observe conflict between quantum theory and noncontextual realistic models [6,14-16]. A recent experiment has demonstrated quantum contextuality for photonic qutrits in a particular quantum state [14], based on a version of the KS inequality proposed by Klyachko et al. [15].

A state-independent test of quantum contextuality for a single qutrit, in the spirit of the original KS theorem, is possible but complicated, as one needs to measure many experimental configurations $[4,6,16]$. A recent theoretical work by $\mathrm{Yu}$ and $\mathrm{Oh}$ proposes another version of the KS inequality, which requires one to measure 13 variables and 24 of their pair correlations [6]. This is a significant simplification compared with the previous KS inequalities for single qutrits, and the number of variables cannot be further reduced as proven recently by Cabello [17]. Our experiment confirms quantum contextuality in a stateindependent fashion using the $\mathrm{Yu}-\mathrm{Oh}$ version of the KS inequality for qutrits represented by three distinctive paths of single photons [18]. The maximum violation of this inequality by quantum mechanics is only $4 \%$ beyond the bound set by the noncontextual hidden variable theory, so we need to accurately control the paths of single photons in experiments to measure the 13 variables and their correlations for different types of input states. We have achieved a violation of the KS inequality by more than 5 standard deviations for all the nine different states that we tested.

For a single qutrit with basis vectors $\{|0\rangle,|1\rangle,|2\rangle\}$, we detect projection operators to the states $i|0\rangle+j|1\rangle+k|2\rangle$ specified by the 13 unit vectors $(i, j, k)$ in Fig. 1 . The 13 projectors have eigenvalues either 0 or 1 . In the hidden variable theory, the corresponding observables are assigned randomly with values 0 or 1 according to a (generally unknown) joint probability distribution. When two states are orthogonal, the projectors onto them commute, and the corresponding observables are called compatible, which means that they can be measured simultaneously. Noncontextuality means that the assignment of values to an observable should be independent of the choice of compatible observables that are measured jointly with it. For instance, $z_{1}$ in Fig. 1 should be assigned the same value in the correlators $z_{1} z_{2}$ and $z_{1} y_{1}^{ \pm}$for each trial of 


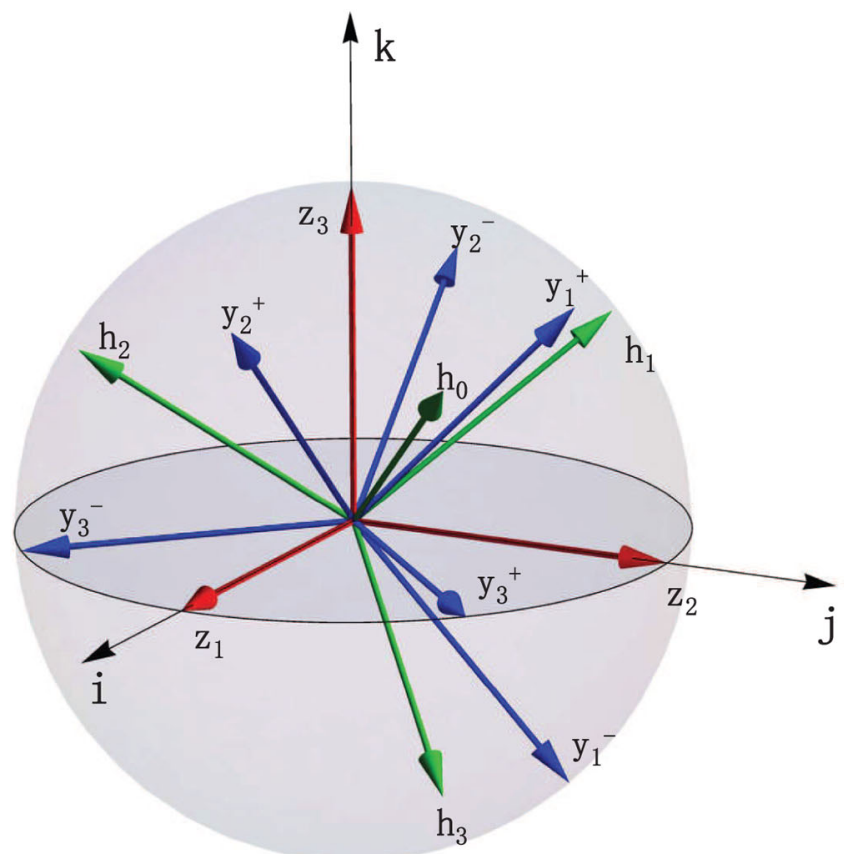

\begin{tabular}{|c|c|}
\hline$z_{1}=(1,0,0)$ & $y_{1}^{-}=\frac{1}{\sqrt{2}}(0,1,-1)$ \\
\hline$z_{2}=(0,1,0)$ & $y_{2}^{-}=\frac{1}{\sqrt{2}}(-1,0,1)$ \\
\hline$z_{3}=(0,0,1)$ & $y_{3}^{-}=\frac{1}{\sqrt{2}}(1,-1,0)$ \\
\hline$h_{1}=\frac{1}{\sqrt{3}}(-1,1,1)$ & $y_{1}^{+}=\frac{1}{\sqrt{2}}(0,1,1)$ \\
\hline$h_{2}=\frac{1}{\sqrt{3}}(1,-1,1)$ & $y_{2}^{+}=\frac{1}{\sqrt{2}}(1,0,1)$ \\
\hline$h_{3}=\frac{1}{\sqrt{3}}(1,1,-1)$ & $y_{3}^{+}=\frac{1}{\sqrt{2}}(1,1,0)$ \\
\hline$h_{0}=\frac{1}{\sqrt{3}}(1,1,1)$ & \\
\hline
\end{tabular}

FIG. 1 (color online). Illustration of the 13 unit vectors that describe the superposition coefficients of 13 corresponding qutrit states. The KS inequality proposed in Ref. [6] requires one to detect projection operators onto these 13 states and their correlations.

measurement. For each observable $b_{i} \in\left\{z_{\mu}, y_{\mu}^{ \pm}, h_{\alpha}, \mu=\right.$ $1,2,3 ; \alpha=0,1,2,3\}$ defined in Fig. 1, we introduce a new variable $a_{i} \equiv 1-2 b_{i}$, which takes values of \pm 1 . For the 13 observables $a_{i}$ with two outcomes \pm 1 , it is shown in Ref. [6] that they satisfy the inequality

$$
\sum_{i} a_{i}-\frac{1}{4} \sum_{\langle i, j\rangle} a_{i} a_{j} \leq 8
$$

where $\langle i, j\rangle$ denotes all pairs of observables that are compatible with each other. There are 24 compatible pairs among all the $13 \times 13$ combinations, and a complete list of them is given in the table in Fig. 2 for the corresponding operator correlations. The inequality (1) can be proven either through an exhaustive check of all the possible $2^{13}$ value assignments of $a_{i}(i=1,2, \ldots, 13)$ or by a more elegant analytic argument as shown in Ref. [6]. In quantum theory, each $a_{i}$ corresponds to an operator $A_{i}$ with

\begin{tabular}{|c|c|c|c|c|c|c|c|c|c|c|}
\hline Observables & \multicolumn{2}{|c|}{$\begin{array}{l}\text { Experimental } \\
\text { value }\end{array}$} & $\begin{array}{l}\text { Theoretical } \\
\text { prediction }\end{array}$ & Observables & \multicolumn{2}{|c|}{$\begin{array}{l}\text { Experimental } \\
\text { value }\end{array}$} & $\begin{array}{l}\begin{array}{l}\text { Theoretical } \\
\text { prediction }\end{array} \\
\end{array}$ & Observables & \begin{tabular}{|l}
$\begin{array}{l}\text { Experimental } \\
\text { value }\end{array}$ \\
\end{tabular} & \begin{tabular}{|l}
$\begin{array}{l}\text { Theoretical } \\
\text { prediction }\end{array}$ \\
\end{tabular} \\
\hline$\left\langle\mathrm{A}_{\mathrm{z}_{1}}\right\rangle$ & \multicolumn{2}{|c|}{$0.328(18)$} & 0.333 & $\left\langle\mathrm{~A}_{\mathrm{z}_{1}} \mathrm{~A}_{\mathrm{z}_{2}}\right\rangle$ & \multicolumn{2}{|c|}{$-0.348(18)$} & -0.333 & $\left\langle\mathrm{~A}_{\mathrm{y}_{1}^{+}} \mathrm{A}_{h_{2}}\right\rangle$ & $-0.628(17)$ & -0.555 \\
\hline$\left\langle\mathrm{A}_{\mathrm{z}_{2}}\right\rangle$ & \multicolumn{2}{|c|}{$0.324(18)$} & 0.333 & $\left\langle\mathrm{~A}_{\mathrm{z}_{\mathrm{z}}} \mathrm{A}_{\mathrm{z}_{\mathrm{z}}}\right\rangle$ & \multicolumn{2}{|c|}{$-0.324(18)$} & -0.333 & $\left\langle\mathrm{~A}_{\mathrm{y}^{+}} \mathrm{A}_{h_{3}}\right\rangle$ & -0.48 & .555 \\
\hline$\left\langle\mathrm{A}_{\mathrm{z}_{3}}\right\rangle$ & \multicolumn{2}{|c|}{$0.348(18)$} & 0.333 & $\left\langle\mathrm{~A}_{\mathrm{z}_{1}} \mathrm{~A}_{\mathrm{z}_{3}}\right\rangle$ & \multicolumn{2}{|c|}{$-0.328(18)$} & -0.333 & $\left\langle\mathrm{~A}_{\mathrm{y}_{2}}-\mathrm{A}_{\mathrm{y}_{2}^{+}}\right\rangle$ & \begin{tabular}{|l|}
$-0.317(17)$ \\
\end{tabular} & \begin{tabular}{|l|}
-0.333 \\
\end{tabular} \\
\hline$\left\langle\mathrm{A}_{\mathrm{y}_{1}^{+}}\right\rangle$ & \multicolumn{2}{|c|}{$-0.320(17)$} & -0.333 & $\left\langle\mathrm{~A}_{\mathrm{z}_{1}} \mathrm{~A}_{\mathrm{y}_{1}^{+}}\right\rangle$ & \multicolumn{2}{|c|}{$-0.876(9)$} & -1 & $\left\langle\mathrm{~A}_{\mathrm{y}_{2}}-\mathrm{A}_{h_{0}}\right\rangle$ & $-0.940(6)$ & \begin{tabular}{|l|}
-1 \\
\end{tabular} \\
\hline$\left\langle\mathrm{A}_{\mathrm{y}_{1}^{-}}\right\rangle$ & \multicolumn{2}{|c|}{$0.876(9)$} & 1 & $\left\langle\mathrm{~A}_{\mathrm{z}_{1}} \mathrm{~A}_{\mathrm{y}_{1}^{-}}\right\rangle$ & \multicolumn{2}{|c|}{$0.320(17)$} & 0.333 & $\left\langle\mathrm{~A}_{\mathrm{y}_{2}} \mathrm{~A}_{h_{1}}\right\rangle$ & $-0.435(16)$ & -0.555 \\
\hline$\left\langle\mathrm{A}_{\mathrm{y}_{2}^{+}}\right\rangle$ & \multicolumn{2}{|c|}{$-0.220(17)$} & -0.333 & $\left\langle\mathrm{~A}_{\mathrm{z}_{2}} \mathrm{~A}_{\mathrm{y}_{2}^{+}}\right\rangle$ & \multicolumn{2}{|c|}{$-0.903(8)$} & -1 & $\left\langle\mathrm{~A}_{\mathrm{y}_{2}}-\mathrm{A}_{h_{2}}\right\rangle$ & $0.682(13)$ & 0.778 \\
\hline$\left\langle\mathrm{A}_{\mathrm{y}_{2}^{-}}\right\rangle$ & \multicolumn{2}{|c|}{$0.903(8)$} & 1 & $\left\langle\mathrm{~A}_{\mathrm{z}_{2}} \mathrm{~A}_{\mathrm{y}_{2}^{-}}\right\rangle$ & \multicolumn{2}{|c|}{$0.220(17)$} & 0.333 & $\left\langle\mathrm{~A}_{\mathrm{y}_{2}^{+}} \mathrm{A}_{h_{3}}\right\rangle$ & $-0.572(15)$ & -0.555 \\
\hline$\left\langle\mathrm{A}_{\mathrm{y}_{3}^{+}}\right\rangle$ & \multicolumn{2}{|c|}{$-0.255(18)$} & -0.333 & $\left\langle\mathrm{~A}_{\mathrm{z}_{3}} \mathrm{~A}_{\mathrm{y}_{3}^{+}}\right\rangle$ & \multicolumn{2}{|c|}{$-0.939(6)$} & -1 & $\left\langle\mathrm{~A}_{\mathrm{y}_{3}^{-}}^{-} \mathrm{A}_{\mathrm{y}_{3}^{+}}\right\rangle$ & -0.31 & -0.333 \\
\hline$\left\langle\mathrm{A}_{\mathrm{y}_{3}^{-}}\right\rangle$ & \multicolumn{2}{|c|}{$0.939(6)$} & 1 & $\left\langle\mathrm{~A}_{\mathrm{z}_{3}} \mathrm{~A}_{\mathrm{y}_{3}^{-}}\right\rangle$ & \multicolumn{2}{|c|}{$0.255(18)$} & 0.333 & $\left\langle\mathrm{~A}_{\mathrm{y}_{3}}-\mathrm{A}_{h_{0}}\right\rangle$ & $-0.979(4)$ & -1 \\
\hline$\left\langle\mathrm{A}_{\mathrm{h}_{0}}\right\rangle$ & \multicolumn{2}{|c|}{$-0.943(6)$} & -1 & $\left\langle\mathrm{~A}_{\mathrm{y}_{1}}-\mathrm{A}_{\mathrm{y}_{1}^{+}}\right\rangle$ & \multicolumn{2}{|c|}{$-0.444(17)$} & -0.333 & $\left\langle\mathrm{~A}_{\mathrm{y}_{3}}+\mathrm{A}_{h_{1}}\right\rangle$ & $-0.480(16)$ & -0.555 \\
\hline$\left\langle\mathrm{A}_{\mathrm{h}_{1}}\right\rangle$ & \multicolumn{2}{|c|}{$0.745(12)$} & 0.778 & $\left\langle\mathrm{~A}_{\mathrm{y}_{1}}-\mathrm{A}_{h_{0}}\right\rangle$ & \multicolumn{2}{|c|}{$-0.938(6)$} & -1 & $\left\langle\mathrm{~A}_{3}^{+} \mathrm{A}_{h_{2}}\right\rangle$ & \begin{tabular}{|c|}
$-0.510(16)$ \\
\end{tabular} & -0.555 \\
\hline$\left\langle\mathrm{A}_{2}\right\rangle$ & \multicolumn{2}{|c|}{$0.817(10)$} & 0.778 & $\left\langle\mathrm{~A}_{\mathrm{y}_{1}}-\mathrm{A}_{h_{1}}\right\rangle$ & \multicolumn{2}{|c|}{$0.698(12)$} & 0.778 & $\left\langle\mathrm{~A}_{\mathrm{y}_{3}}-\mathrm{A}_{h_{3}}\right\rangle$ & $0.710(13)$ & 0.778 \\
\hline$\left\langle\mathrm{A}_{\mathrm{h}_{3}}\right\rangle$ & \multicolumn{2}{|c|}{$0.746(12)$} & 0.778 & & & & & & & \\
\hline \multicolumn{2}{|c|}{ K-S Inequality (2) } & \multicolumn{4}{|c|}{$\sum\left\langle A_{i}\right\rangle-\frac{1}{4} \sum_{\langle i, j\rangle}\left\langle A_{i} A_{j}\right\rangle=8.224 \pm 0.041$} & \multicolumn{2}{|c|}{ K-S Inequality (3) } & \multicolumn{3}{|c|}{$\sum\left\langle B_{j}\right\rangle=1.317 \pm 0.011$} \\
\hline
\end{tabular}

FIG. 2. The measured expectation values $\left\langle A_{i}\right\rangle$ and the correlations $\left\langle A_{i} A_{j}\right\rangle$ for all the compatible pairs under a particular input state $|s\rangle=(|0\rangle+|1\rangle+|2\rangle) / \sqrt{3}$. For the experimental values, the numbers in the bracket represent the statistical error associated with the photon detection under the assumption of a Poissonian distribution for the photon counts, for instance, $\left\langle A_{z_{1}}\right\rangle=0.328(18) \equiv$ $0.328 \pm 0.018$. Both of the inequalities (2) and (3) are significantly violated by the experimental data. 
eigenvalues \pm 1 . In the hidden variable theory, the value $a_{i}$ corresponds to a random variable $A_{i}$, and the different values are distributed according to a (possibly correlated) joint probability distribution. Hence, for the hidden variable theory the expectation values of $A_{i}$ must satisfy the inequality

$$
\sum_{i}\left\langle A_{i}\right\rangle-\frac{1}{4} \sum_{\langle i, j\rangle}\left\langle A_{i} A_{j}\right\rangle \leq 8
$$

which follows by taking the average of (1) over the joint probability distribution of the values $a_{i}$. On the other hand, quantum theory gives a different prediction: From the definition $A_{i} \equiv I-2 B_{i}$, where $B_{i}$ is the projection operator to the 13 states in Fig. 1, we find that $S=\sum_{i} A_{i}-$ $\frac{1}{4} \sum_{\langle i, j\rangle} A_{i} A_{j} \equiv \frac{25}{3} I$, where $I$ is the unity operator. Hence, for any state of the system, quantum theory predicts the inequality $\langle S\rangle=25 / 3 \not \leq 8$, which violates the inequality (2) imposed by the noncontextual hidden variable theory and rules out any noncontextual realistic model.

Since the quantum mechanical prediction $\langle S\rangle=25 / 3$ is close to the upper bound $\langle S\rangle \leq 8$ set by the noncontextual realism, we need to achieve accurate control in experiments to violate the inequality (2). Yu and $\mathrm{Oh}$ also derived another simpler inequality in Ref. [6] by introducing an additional assumption (as proposed in the original KS proof $[1,4])$ that the algebraic structure of compatible observables is preserved at the hidden variable level, that is, that the value assigned to the product (or sum) of two compatible observables is equal to the product (or sum) of the values assigned to these observables. Under this assumption, it is shown in Ref. [6] that

$$
\sum_{\alpha=0,1,2,3}\left\langle B_{h_{\alpha}}\right\rangle \leq 1
$$

for the noncontextual hidden variable theory, while quantum mechanically $\sum_{\alpha=0,1,2,3} B_{h_{\alpha}} \equiv \frac{4}{3} I$, and thus $\sum_{\alpha=0,1,2,3}\left\langle B_{h_{\alpha}}\right\rangle=4 / 3>1$. The inequality (3) is more amenable to experimental tests than Eq. (2), as it requires only four measurement settings. However, conceptually it is weaker than Eq. (2) due to the additional assumption required for its proof. Our experiment achieves significant violation of both the inequalities (2) and (3).

To experimentally test the inequalities (2) and (3), first we prepare a single photonic qutrit through the spontaneous parametric down-conversion setup shown in Fig. 3. The spontaneous parametric down-conversion process generates correlated (entangled) photon pairs, and, through detection of one of the photons by a detector D0, we get a

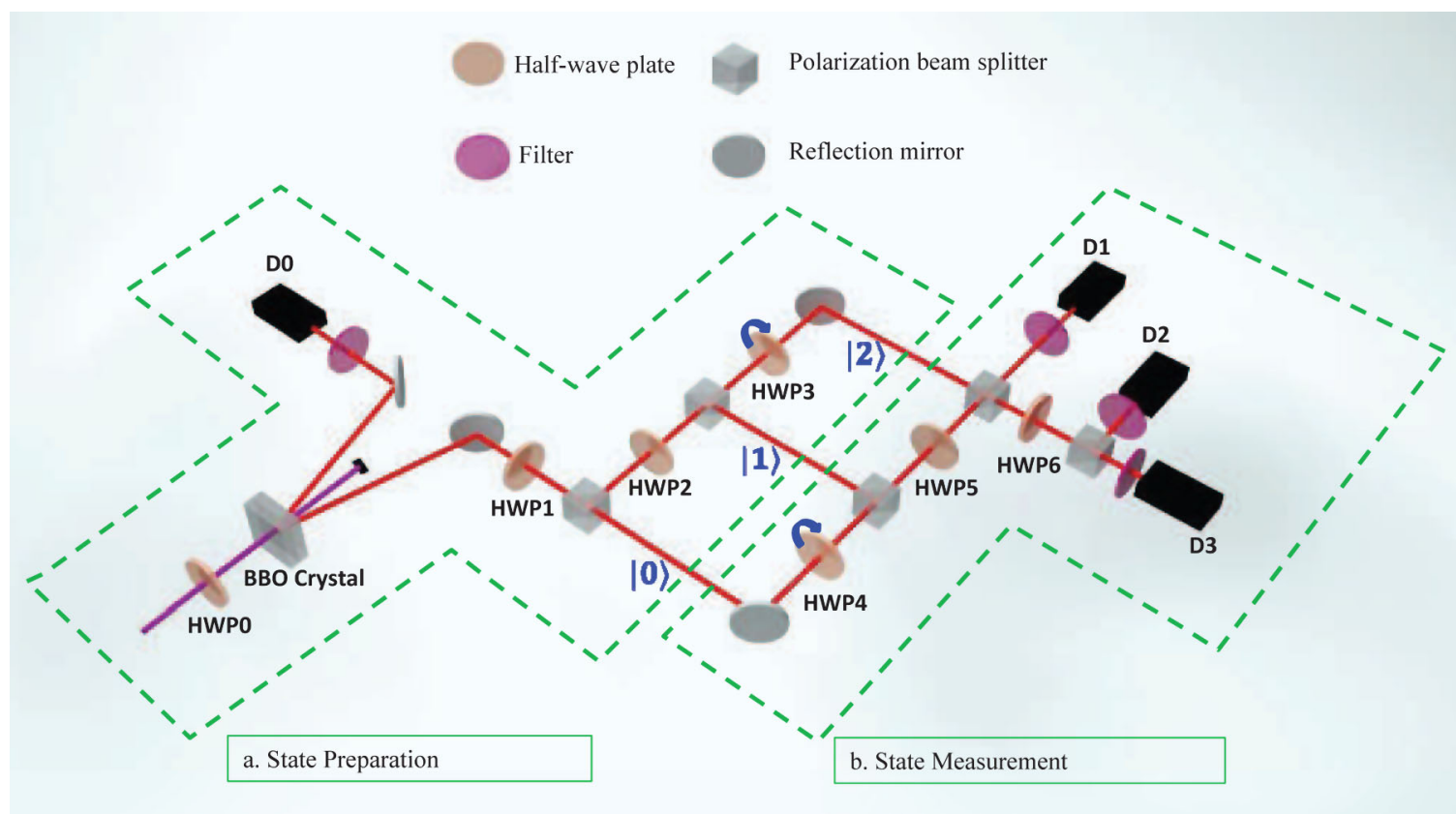

FIG. 3 (color online). Illustration of the experimental setup to detect the KS inequalities. The setup in box (a) is for state preparation of a single photonic qutrit. Ultrafast laser pulses (with a repetition rate of $76 \mathrm{MHz}$ ) at the wavelength of $400 \mathrm{~nm}$ from a frequency doubled Ti:sapphire laser pump two joint beta-barium-borate crystals, each of $0.6 \mathrm{~mm}$ depth with a perpendicular optical axis, to generate correlated (entangled) photon pairs at the wavelength of $800 \mathrm{~nm}$. With registration of a photon count at the detector D0, we get a heralded single-photon source in the other output mode. This photon is split by two PBSs into three optical paths, representing a single photonic qutrit. By adjusting the angle of the half wave plates (HWP1 and HWP2), we can control the superposition coefficients of this qutrit state. The setup in box (b) is for measurement of the qutrit state along compatible projections to three orthogonal states. By tuning the wave plates (HWP5 and HWP6), we choose these projection operators to be along the directions specified by the $A_{i}$ operators to measure the correlations of the compatible $A_{i}$. The wave plates HWP3 and HWP4 are used to balance the Mach-Zehnder interferometers and can be tilted for fine-tuning of the relative phase. 
heralded single-photon source on the other output mode. This photon is then split by two polarizing beam splitters (PBSs) into three spatial modes that represent a single photonic qutrit. Through control of the wave plates before the PBSs and for the pump light, we can prepare any state for this photonic qutrit.

The state of the qutrit is then detected by three singlephoton detectors D1-D3. To measure the observables $A_{i}$ and their correlations, we use the setup shown in Fig. 1 based on cascaded Mach-Zehnder interferometers. The wave plates HWP3 and HWP4 in the interferometers can be tilted to fine-tune the phase difference between the two arms. To stabilize the relative phase, the whole interferometer setup is enclosed in a black box. The detectors D1-D3 measure projections to three orthogonal states in the qutrit space, which always correspond to mutually compatible observables. By tuning the half wave plates HWP5 and HWP6 in Fig. 1, we can choose these projections so that they give a subset of the 13 projection operators $B_{i}$. A detector click (nonclick) then means assignment of value $1(0)$ to the corresponding observable $B_{i}$ [or, equivalently, assignment of $-1(+1)$ to the observable $\left.A_{i}\right]$. The coincidence between the detectors measures the correlation. The detailed configurations of the wave plates to measure different correlations are summarized in Sec. I of the Supplemental Material [19]. Because of the photon loss, sometimes our photonic qutrit does not yield a click in the detectors D1-D3, even though we registered a heralding photon at the detector D0. To take this into account, we discard the events when none of the detectors D1-D3 fires, in the same way as it was done in Ref. [14]. The use of this postselection technique opens up a detection efficiency loophole, and we need to assume that the events selected out by the photonic coincidence are an unbiased representation of the whole sample (fair-sampling assumption).

We have measured all the expectation values in the inequality (2) and (3) for different input states. The table in Fig. 2 summarizes the measurement results for a particular input state $|s\rangle=(|0\rangle+|1\rangle+|2\rangle) / \sqrt{3}$ in equal superposition of the three basis vectors. The theoretical values in the quantum mechanical case are calculated by using the Born rule with the ideal state $|s\rangle$. Each of the experimental correlations is constructed from the joint probabilities $P\left(A_{i}= \pm 1 ; A_{j}= \pm 1\right)$ registered by the detectors. As an example to show the measurement method, in Sec. II of the Supplemental Material [19], we give detailed data for the registered joint probabilities under different measurement configurations, which together fix all the correlations in the table in Fig. 2. The expectation value $\left\langle B_{i}\right\rangle$ (or $\left.\left\langle A_{i}\right\rangle \equiv 1-2\left\langle B_{i}\right\rangle\right)$ is directly determined by the relative probability of the photon firing in the corresponding detector. From the data summarized in the table in Fig. 2, we find that both of the inequalities (2) and (3) are significantly violated in experiments, in agreement with the quantum mechanics prediction and in contradiction with the noncontextual realistic models. Even the tough inequality (2) is violated by more than 5 times the error bar (standard deviation).

To verify that the inequalities (2) and (3) are experimentally violated independently of the state of the system, we have tested them for different kinds of input states. The set of states tested include the three basis vectors $\{|0\rangle,|1\rangle,|2\rangle\}$, the two-component superposition states $\{(|0\rangle+|1\rangle) / \sqrt{2}$, $(|0\rangle+|2\rangle) / \sqrt{2},(|1\rangle+|2\rangle) / \sqrt{2}\}$, the three-component superposition state $|s\rangle$, and two mixed states $\rho_{8}=(|0\rangle\langle 0|+$ $|2\rangle\langle 2|) / 2 \quad$ and $\quad \rho_{9}=(|0\rangle\langle 0|+| 1\rangle\langle 1|+| 2\rangle\langle 2|) / 3 \equiv I / 3$. The detailed configurations of the wave plates to prepare these different input states are summarized in Sec. I of the Supplemental Material [19]. To generate the mixed states, we first produce photon pairs entangled in polarization by using the type-I phase matching in the beta-barium-borate crystal [20]. After tracing out the idler photon by the detection at D0, we get a mixed state in polarization for the signal photon, which is then transferred to a mixed qutrit state represented by the optical paths through the PBS. For various input states, we measure correlations of all the observables in the inequality (2), and the detailed results are presented in Sec. III of the Supplemental Material [19]. Although the expectation values $\left\langle A_{i}\right\rangle$ and the correlations $\left\langle A_{i} A_{j}\right\rangle$ strongly depend on the input states, the inequalities (2) and (3) are state-independent and significantly violated for all the cases tested in experiments. In Fig. 4, we present the measurement outcomes of these two inequalities for nine different input states. The results clearly violate the boundary set by the noncontextual hidden variable theory.

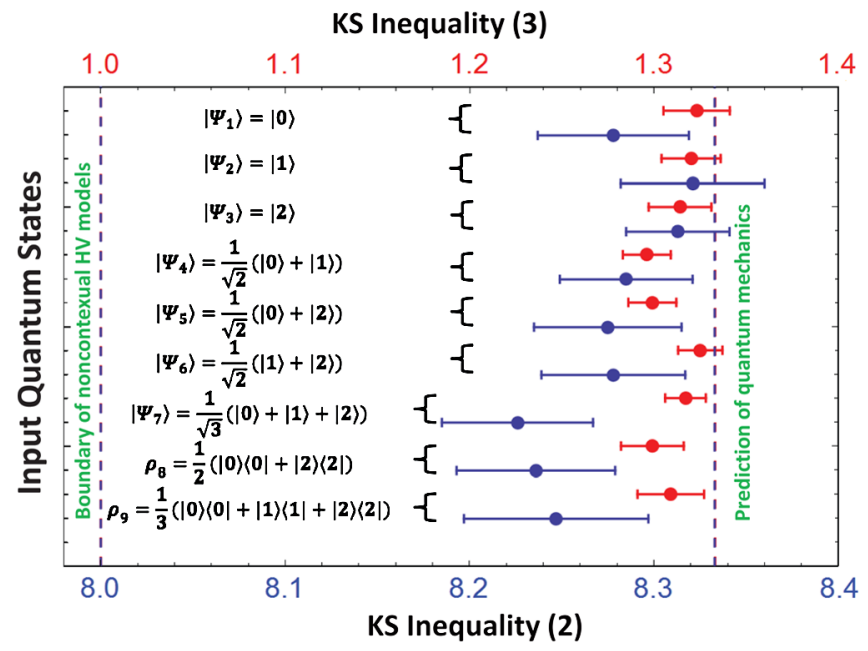

FIG. 4 (color online). The measurement results for the two inequalities (2) (shown by the blue label) and (3) (shown by the red label) under different types of input states. The left-side dashed line specifies the upper bounds imposed by any noncontextual hidden variable models, while the right-side dashed line corresponds to the quantum mechanical prediction under the ideal input states. The error bars account for the statistical error associated with the photon detection. 
In this work, we have observed violation of the $\mathrm{KS}$ inequalities (2) and (3) for a single photonic qutrit, which represents the first state-independent experimental test of quantum contextuality in an indivisible quantum system. The experiment confirmation of quantum contextuality in its most basic form, in a way that is independent of either the state or the tensor product structure of the system, sheds new light on the contradiction between quantum mechanics and noncontextual realistic models.

This work was supported by the National Basic Research Program of China (973 Program) 2011CBA00300 (2011CBA00302) and the NSFC Grant 61033001. D.-L. D. and L.-M. D. acknowledge in addition support from the IARPA MUSIQC program, the ARO, and the AFOSR MURI program.

[1] S. Kochen and E. P. Specker, J. Math. Mech. 17, 59 (1967).

[2] J. S. Bell, Rev. Mod. Phys. 38, 447 (1966).

[3] N. D. Mermin, Phys. Rev. Lett. 65, 3373 (1990).

[4] A. Peres, Quantum Theory: Concepts and Methods (Kluwer, Dordrecht, 1993), Chap. 7.

[5] A. Cabello, Phys. Rev. Lett. 101, 210401 (2008).

[6] S. X. Yu and C. H. Oh, Phys. Rev. Lett. 108, 030402 (2012).

[7] D. A. Meyer, Phys. Rev. Lett. 83, 3751 (1999).

[8] A. Kent, Phys. Rev. Lett. 83, 3755 (1999).
[9] G. Kirchmair, F. Zähringer, R. Gerritsma, M. Kleinmann, O. Gühne, A. Cabello, R. Blatt, and C.F. Roos, Nature (London) 460, 494 (2009).

[10] E. Amselem, M. Radmark, M. Bourennane, and A. Cabello, Phys. Rev. Lett. 103, 160405 (2009).

[11] Y.-F. Huang, C.-F. Li, Y.-S. Zhang, J.-W. Pan, and G.-C. Guo, Phys. Rev. Lett. 90, 250401 (2003).

[12] H. Bartosik, J. Klepp, C. Schmitzer, S. Sponar, A. Cabello, H. Rauch, and Y. Hasegawa, Phys. Rev. Lett. 103, 040403 (2009).

[13] O. Moussa, C. A. Ryan, D. G. Cory, and R. Laflamme, Phys. Rev. Lett. 104, 160501 (2010).

[14] R. Lapkiewicz, P. Li, C. Schaeff, N. K. Langford, S. Ramelow, M. Wieśniak, and A. Zeilinger, Nature (London) 474, 490 (2011).

[15] A. A. Klyachko, M. A. Can, S. Binicioglu, and A. S. Shumovsky, Phys. Rev. Lett. 101, 020403 (2008).

[16] P. Badziag, I. Bengtsson, A. Cabello, and I. Pitowsky, Phys. Rev. Lett. 103, 050401 (2009).

[17] A. Cabello, arXiv:1112.5149.

[18] A. Cabello, E. Amselem, K. Blanchfield, M. Bourennane, and I. Bengtsson, Phys. Rev. A 85, 032108 (2012).

[19] See Supplemental Material at http://link.aps.org/ supplemental/10.1103/PhysRevLett.109.150401 for detailed data and further description of the experimental configuration.

[20] P. G. Kwiat, E. Waks, A. G. White, I. Appelbaum, and P. H. Eberhard, Phys. Rev. A 60, R773 (1999). 